\title{
Tibial Accelerations During the Single-Leg Hop Test: Influence of Fixation
}

\author{
Hannah W. Tucker, Emily R. Tobin, and Matthew F. Moran
}

\begin{abstract}
Context: Performance on single-leg hopping (SLH) assessments is commonly included within return-to-sport criteria for rehabilitating athletes. Triaxial accelerometers have been used to quantify impact loading in a variety of movements, including hopping; however, they have never been attached to the tibia during SLH, and their method of fixation has not been investigated. Objective: The purpose of this study was to quantify triaxial accelerations and evaluate the influence of the fixation method of a lightweight inertial measurement unit (Blue Trident) mounted to the tibia during SLH performance. Design: Single cohort, repeated-measures experimental design. Participants: Sixteen healthy participants (10 females and 6 males; 20 [0.9] y; $1.67[0.08] \mathrm{m} ; 66.0$ [8.5] kg) met the inclusion criteria, volunteered, and completed this study. Interventions: Participants performed 2 sets of $3 \mathrm{SLH}$ trials with an inertial measurement unit $(1500 \mathrm{~Hz})$ fixated to the tibia, each set with 1 of 2 attachment methods (double-sided tape [DST] with athletic tape and silicon strap [SS] with Velcro adhesion). Main Outcome Measures: Hop distance, peak tibial acceleration (PTA), time to PTA, and the acceleration slope were assessed during each hop landing. Results: Repeated-measures analysis of variance determined no significant effect of the attachment method on hop metrics $(P=.252)$. Across 3 trials, both fixation methods (DST and SS) had excellent reliability values (intraclass correlation coefficient: .868-.941) for PTA and acceleration slope but not for time to PTA (intraclass correlation coefficient: .397-.768). The PTA for DST (27.22 [7.94] $g$ ) and SS (26.21 [10.48] $g$ ) was comparable and had a moderate, positive relationship (DST: $r=.72, P<.01$; SS: $r=.77, P<.01$ ) to SLH distance. Conclusions: Tibial inertial measurement units with triaxial accelerometers can reliably assess PTA during performance of the SLH, and SS is a viable alternative tibial attachment to DST.
\end{abstract}

Keywords: hopping, return to sport, IMU

Anterior cruciate ligament (ACL) tears are a common injury with up to 200,000 ACL reconstruction (ACL-R) surgical procedures performed annually. ${ }^{1}$ For an ACL-R athlete, sports medicine professionals determine eligibility for return to sport (RTS) based on several factors. Hopping assessments, specifically single-leg maximal hop and hold (SLH), have commonly been included..$^{2-4}$ Body-worn inertial measurement units (IMUs) have been utilized to establish a strong correlation between tibial acceleration and peak landing forces during a vertical jump ${ }^{5}$; however, only one study has reported on their use during SLH. ${ }^{6}$ Using a trunkmounted accelerometer via double-sided tape (DST), Williams et $\mathrm{al}^{6}$ reported that a measure of postural sway to quantify hop landing balance had moderate-to-excellent reliability. ${ }^{6}$

The integrity of fixation between accelerometer and body influences the measurement signal because of noise introduced from device resonance. ${ }^{7}$ As the vertical ground reaction forces are considerably larger during hopping ${ }^{8}$ than running, it is unclear if these large landing forces would cause device oscillation inducing an increased and faulty acceleration magnitude. To mitigate measurement noise, the most accurate fixation method uses intracortical bone pins ${ }^{9}$; however, this method is not feasible from a sports medicine perspective. With appropriate fixation and digital filtering, the acceleration signal from a skin-mounted device adequately corroborates with actual bony accelerations. ${ }^{9}$ The majority of reports have fixated accelerometers through some application of

The authors are with the Department of Physical Therapy and Human Movement Science, Sacred Heart University, Fairfield, CT, USA. Moran (moranm@ sacredheart.edu) is corresponding author. double-sided tape ${ }^{10}$ or elastic bands. ${ }^{7}$ Currently, there are no studies that have analyzed the influence of fixation method on acceleration magnitudes during the SLH landing phase.

Therefore, the purpose of this study was to evaluate the influence of fixation method of a lightweight IMU device mounted to the tibia during SLH landing. The most commonly reported fixation method (DST) was compared with a silicon strap with Velcro adhesion (SS). It was hypothesized that SS would produce comparable acceleration magnitudes to DST fixation.

\section{Methods}

\section{Design}

A single cohort, repeated-measures design was used to investigate the relationship among hop distance, peak tibial acceleration (PTA), time to PTA and peak tibial acceleration slope (AS; dependent variables), and tibial IMU fixation method (DST and SS; independent variable) during performance of the SLH.

\section{Participants}

Participants were free of any current or previous lower-extremity injuries in which medical care was sought, and they had also engaged in regular exercise for the previous 3 consecutive months. Testing procedures were approved by the Sacred Heart University's institutional review board. All participants ( 10 females and 6 males; 20 [0.9] y; 1.67 [0.08] m; 66.0 [8.5] kg) signed a health history form and provided informed consent. 


\section{Procedures}

A lightweight (9.5 $\mathrm{g}$ ) IMU (Blue Trident; Vicon, Auckland, New Zealand) was utilized; it featured both low- $g( \pm 16 g)$ and high- $g$ $( \pm 200 \mathrm{~g})$ triaxial accelerometers. Measurement tape was used to assess ankle circumference and mark a location $3 \mathrm{~cm}$ proximal to medial malleolus. ${ }^{10}$ Two fixation methods were studied as follows: (1) $\mathrm{DST}^{10}$ and (2) manufacturer-supplied $\mathrm{SS}^{11}$ (Vicon; Figure 1).

Anthropometric measures (height, weight, and ankle circumference) were collected, including leg dominance, which was defined as the leg used to kick a ball. ${ }^{2}$ The participants completed a standardized warm-up (jumping jacks and bilateral walking lunges) before being given a verbal and visual SLH demonstration. Specifically, the participants were instructed to hop as far forward as possible and hold the landing position for approximately 3 seconds while maintaining their arms behind their back.

The IMU device was attached to the dominant leg in a counterbalanced order. Ankle circumference thresholds (small: $\leq 19 \mathrm{~cm}$, medium: 19-24 cm, and large: $\geq 24 \mathrm{~cm}$ ) were utilized for proper fitting of SS, which was iteratively tightened as "tightly as tolerable" by the participant via Velcro adhesion (Figure 1).

Following 2 SLH practice trials, the participants completed 3 trials while maintaining their hands behind their back ${ }^{12}$ and holding the final landing position for approximately 3 seconds. Hop distance was measured for each trial, and 30 seconds of rest was granted between trials. Participants had 5 minutes of rest between the attachment method conditions. Data collection was initiated using the Capture.U application (1500 Hz; Vicon).

A MATLAB script (MathWorks, Natick, MA) filtered acceleration data (x, y, and $\mathrm{z}$ axes) with a $60-\mathrm{Hz}$, fourth-order, dual-pass Butterworth low-pass filter before resultant acceleration was computed as $r=\left(x^{2}+y^{2}+z^{2}\right)^{0.5} \cdot{ }^{10}$ The resultant acceleration eliminated potential error associated with device orientation relative to the long axis of the tibia. ${ }^{7}$ Peak tibial acceleration, time to PTA, and peak tibial AS were determined for each trial based on a previously reported method (Figure 2). ${ }^{13}$ Peak tibial AS was calculated from $30 \%$ to $70 \%$ of the total time to reach PTA.

\section{Statistical Analysis}

Descriptive statistics were calculated for all dependent variables from the maximal hop distance trial from both fixation conditions.

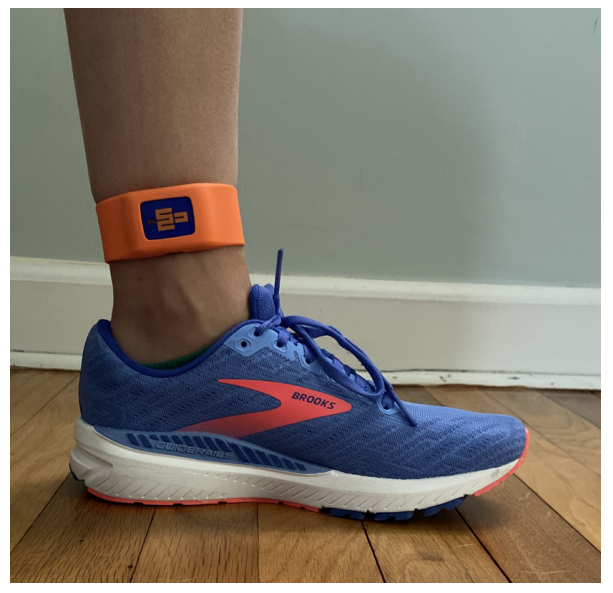

Figure 1 - A lightweight $(9.5 \mathrm{~g})$ and portable inertial measurement unit (Blue Trident) with triaxial accelerometers was attached to the distal tibia $3 \mathrm{~cm}$ proximal to the medial malleolus with silicon straps and Velcro adhesion.
Repeated-measures analysis of variance statistical tests assessed differences in hop metrics based on fixation method. Bonferroni pairwise comparisons post hoc tests were performed to evaluate the effect of the fixation method. The significance level was set a priori at $\alpha=.05$. The effect size was determined by the partial eta-squared $\left(\eta_{p}^{2}\right)$ term.

Test-retest reliability was assessed using 2-way mixed-effects intraclass correlation coefficients $\left(\mathrm{ICC}_{3, k}\right)$. An ICC value $\geq .9$ was considered a "high" level of agreement, $\leq 0.7$ was "poor," and between 0.7 and 0.9 was "questionable." The standard error of the mean and minimal detectable change $\left(\mathrm{MDC}_{95 \%}\right)$ were used to assess natural trial variability for both attachment methods. To explore the relationship between hop distance and PTA, a Pearson correlation analysis was performed on the maximal hop distance trial. All statistical analysis was performed in SPSS software (version 25.0; IBM Corp, Armonk, NY).

\section{Results}

Descriptive statistics for all dependent variables are provided in Table 1. Mauchly's test of sphericity indicated that the sphericity assumption was violated $(P<.05)$; therefore, a GreenhouseGeisser adjustment was used. Repeated-measures analysis of variance based on attachment method revealed no significant main effect $\left(F_{1,15}=1.42, P=.252, \eta_{p}^{2}=.086\right)$ and no significant interaction effect $\left(F_{1,15.002}=1.46, P=.246, \eta_{p}^{2}=.089\right)$.

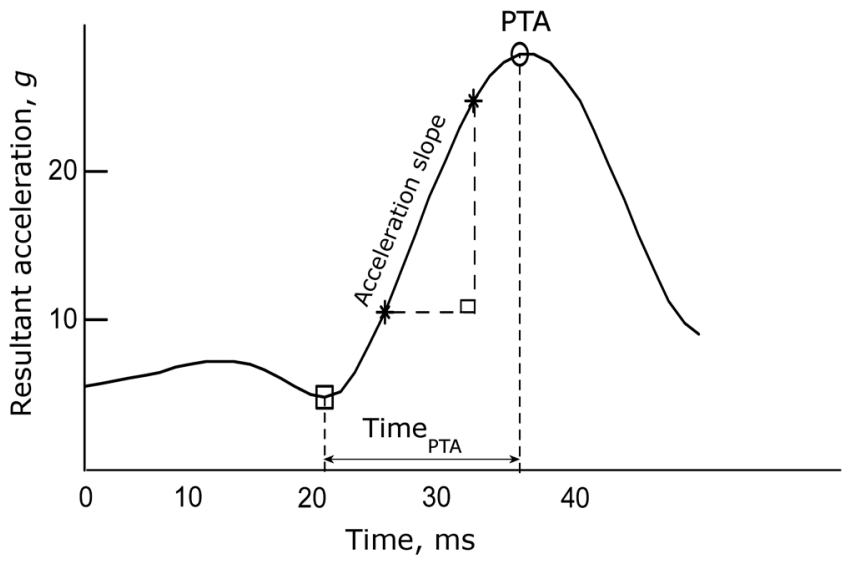

Figure 2 - Representative resultant acceleration data for a participant during the landing phase of the single-leg hop. Three dependent variables were determined for each trial: PTA, time to PTA, and acceleration slope. ${ }^{13}$ PTA indicates peak tibial acceleration.

\section{Table 1 Descriptive Statistics for SLH Test With Both Fixation Methods}

\begin{tabular}{lccccc}
\hline & \multicolumn{2}{c}{ DST $(\mathbf{n = 1 6 )}$} & & \multicolumn{2}{c}{ SS $(\mathbf{n}=\mathbf{1 6})$} \\
\cline { 2 - 3 } \cline { 6 - 6 } Hop Metrics & Mean & SD & & Mean & SD \\
\hline Hop distance, $\mathrm{cm}$ & 102.9 & 24.9 & & 103.8 & 25.5 \\
PTA, $g$ & 27.22 & 7.94 & & 26.21 & 10.48 \\
Time to PTA, ms & 9.50 & 2.48 & & 10.17 & 2.54 \\
AS, $g \cdot \mathrm{ms}^{-1}$ & 2846.53 & 1131.95 & & 2505.19 & 1585.72 \\
\hline
\end{tabular}

Abbreviations: AS, acceleration slope; DST, double-sided tape; PTA, peak tibial acceleration; SLH, single-leg hop; SS, silicon straps. 
Both fixation methods had "high" ICC values (DST $=.916$ and $\mathrm{SS}=.936$ ) for PTA (Table 2). At the $95 \%$ confidence level, the MDC for PTA was 5.7 and $6.6 \mathrm{~g}$, respectively, for the DST and SS methods. These values represent $22.0 \%$ and $25.2 \%$ of the 3 trial mean. For peak tibial AS, the SS fixation method produced more reliable results $(0.941)$ versus DST $(0.868)$. At the $95 \%$ confidence level, MDC for AS was 802.8 and $978.4 \mathrm{~g} \cdot \mathrm{ms}^{-1}$, respectively, for the DST and SS methods. These values represent $31.6 \%$ and $38.8 \%$ of the 3 trial mean. The only hopping metric wherein fixation methods substantially differed in ICC values was time to PTA. The DST produced poor reliability (0.397) and SS produced questionable reliability (0.768).

The relationship of maximal hop distance to PTA demonstrated a significant moderate positive correlation for both attachment methods (DST: $r=.72, P<.01$ and SS: $r=.77, P<.01$ ).

\section{Discussion}

The results of the current study demonstrate that both fixation methods produce comparable acceleration metrics during SLH. The main advantage of utilizing SS versus DST when attaching a wearable tibial IMU device is in the ease of use. Once the SS is sized appropriately, the device can more easily be tightened by the athlete and does not require additional materials.

Although maximal SLH distance is typically utilized to aid in RTS decision making, most protocols involve repeated SLH trials. When analyzing across all trials, the statistical analysis revealed that both fixation methods had similar "high" reliability for PTA with a low standard error of the mean (DST $=7.9 \%$ and $\mathrm{SS}=9.1 \%$ of mean). This corroborated the 1-week and 6-month excellent reliability in detecting PTA during running with the same device ${ }^{10}$ and represented improved reliability than that reported for detecting the landing hop phase with a trunk accelerometer. ${ }^{6}$ Interestingly, the reliability of SS fixation was slightly improved for determining the peak tibial AS than DST. One possible explanation for this difference is that the SS Velcro may allow the device to be iteratively adjusted to tolerance, which is more difficult than DST. Time to PTA produced either poor (DST) or questionable reliability (SS) obviating its potential use in assessing SLH landing with

\section{Table 2 Reliability and Variability of SLH Metrics With} 2 Attachment Methods

\begin{tabular}{|c|c|c|c|}
\hline Hop Metrics & ICC & SEM (\%Mean) & MDC $_{95 \%}(\%$ Mean) \\
\hline \multicolumn{4}{|c|}{ Hop distance, $\mathrm{cm}$} \\
\hline DST & .976 & $6.1(6.3 \%)$ & $16.8(17.4 \%)$ \\
\hline SS & .973 & $6.1(6.2 \%)$ & $16.8(17.3 \%)$ \\
\hline \multicolumn{4}{|l|}{ PTA, $g$} \\
\hline DST & .916 & $2.1(7.9 \%)$ & $5.7(22.0 \%)$ \\
\hline SS & .936 & $2.4(9.1 \%)$ & $6.6(25.2 \%)$ \\
\hline \multicolumn{4}{|c|}{ Time to PTA, ms } \\
\hline DST & .397 & $0.79(7.5 \%)$ & $2.18(20.7 \%)$ \\
\hline SS & .768 & $0.58(5.7 \%)$ & $1.6(15.9 \%)$ \\
\hline \multicolumn{4}{|l|}{ AS, $g / \mathrm{ms}$} \\
\hline DST & .868 & $289.6(11.4 \%)$ & $802.8(31.6 \%)$ \\
\hline SS & .941 & $352.9(14.0 \%)$ & $978.4(38.8 \%)$ \\
\hline
\end{tabular}

this device. Based on the current results, PTA is a reliable metric to monitor during the SLH landing phase.

When analyzing across all participants, the mean PTA was 27.22 (7.94) $g$ for DST and 26.21 (10.48) $g$ for SS (range: 12.6$53.5 \mathrm{~g}$ ), which is substantially higher than for running (7.8$12.9 \mathrm{~g}) .{ }^{10}$ Although this is not surprising given the increased vertical ground reaction forces during SLH, it does highlight the necessity of an increased accelerometer operating range for assessing SLH. Mitschke et $\mathrm{al}^{14}$ reported that the accelerometer operating range $( \pm 8, \pm 16, \pm 32$, and $\pm 70 \mathrm{~g})$ significantly underestimated PTA during running when the operating range was less than $\pm 32 \mathrm{~g}$. The device used in this study had an operating range of $\pm 500 \mathrm{~g}$, which was approximately 9.4 times the maximum PTA.

In the current study, Pearson correlation analysis revealed a positive, moderate relationship with approximately $72 \%$ to $77 \%$ of PTA variance explained by SLH distance. This demonstrates that PTA during the SLH may provide meaningful quantification of the landing phase beyond just monitoring hop distance; however, the MDC of PTA was $22.0 \%$ and $25.2 \%$ of the mean value, respectively, for the DST and SS attachment methods. These statistics should be considered when determining RTS thresholds for bilateral comparisons of the PTA during the SLH. With reinjury rates between $15 \%$ and $23 \%$, an enhanced ability to easily quantify and assess movement quality may aid the sports medicine professional in RTS decision making and potentially lower reinjury rates. ${ }^{15}$ Future studies should expand upon this work by assessing normative bilateral PTA during the SLH and, subsequently, utilize this device to monitor these accelerations in ACL-R individuals postsurgery to determine its potential as an RTS criteria.

\section{Conclusions}

The current study determined that a wearable tibial accelerometer attached via SS can reliably measure PTA and can be used as a viable alternative to DST and elastic bandages. Assessment of PTA can potentially explain the differences of landing mechanics beyond hop distance. Clinicians should consider utilizing wearable tibial accelerometers to monitor rehabilitating athletes and to determine the efficacies of treatment protocols.

\section{Acknowledgments}

The authors report no declaration of interests.

\section{References}

1. Barber-Westin SD, Noyes FR. Factors used to determine return to unrestricted sports activities after anterior cruciate ligament reconstruction. Arthrosc J Arthrosc Relat Surg. 2011;27(12):1697-1705. doi:10.1016/j.arthro.2011.09.009

2. Dingenen B, Truijen J, Bellemans J, Gokeler A. Test-retest reliability and discriminative ability of forward, medial and rotational single-leg hop tests. Knee. 2019;26(5):978-987. PubMed ID: 31431339 doi:10. 1016/j.knee.2019.06.010

3. Forner-Cordero A, Mateu-Arce M, Forner-Cordero I, Alcántara E, Moreno JC, Pons JL. Study of the motion artefacts of skin-mounted inertial sensors under different attachment conditions. Physiol Meas. 2008;29(4):N21-N31. doi:10.1088/0967-3334/29/4/N01

4. Raper DP, Witchalls J, Philips EJ, Knight E, Drew MK, Waddington G. Use of a tibial accelerometer to measure ground reaction force in running: a reliability and validity comparison with force plates. $J \mathrm{Sci}$ 
Med Sport. 2018;21(1):84-88. PubMed ID: 28663135 doi:10.1016/j. jsams.2017.06.010

5. Elvin NG, Elvin AA, Arnoczky SP. Correlation between ground reaction force and tibial acceleration in vertical jumping. $J$ Appl Biomech. 2007;23(3):180-189. PubMed ID: 18089915 doi:10.1123/ jab.23.3.180

6. Williams JM, Gara M, Clark C. The quantification of hop landing balance using trunk-mounted accelerometry. J Sport Rehabil. 2019; 28(8). doi:10.1123/jsr.2018-0384

7. Sheerin KR, Reid D, Besier TF. The measurement of tibial acceleration in runners-a review of the factors that can affect tibial acceleration during running and evidence-based guidelines for its use. Gait Posture. 2019;67:12-24. PubMed ID: 30248663 doi:10.1016/j. gaitpost.2018.09.017

8. Orishimo KF, Kremenic IJ, Mullaney MJ, McHugh MP, Nicholas SJ. Adaptations in single-leg hop biomechanics following anterior cruciate ligament reconstruction. Knee Surg Sports Traumatol Arthrosc. 2010;18(11):1587-1593. PubMed ID: 20549185 doi:10.1007/ s00167-010-1185-2

9. Lafortune MA, Henning E, Valiant GA. Tibial shock measured with bone and skin mounted transducers. J Biomech. 1995;28(8):989-993. PubMed ID: 7673266 doi:10.1016/0021-9290(94)00150-3

10. Sheerin K, Besier T, Reid D, Patria H. The one-week and six-month reliability and variability of three-dimensional tibial acceleration in runners. Sports Biomech. 2017;17(4):1-10. doi:10.1080/14763141. 2017.1371214

11. Tenforde AS, Hayano T, Jamison ST, Outerleys J, Davis IS. Tibial acceleration measured from wearable sensors is associated with loading rates in injured runners. $P M R$. October 2020;12(7):679-684. doi:10.1002/pmrj.12275

12. Gustavsson A, Neeter C, Thomeé $\mathrm{P}$, et al. A test battery for evaluating hop performance in patients with an ACL injury and patients who have undergone ACL reconstruction. Knee Surg Sports Traumatol Arthrosc. 2006;14(8):778-788. doi:10.1007/s00167-0060045-6

13. Duquette AM, Andrews DM. Tibialis anterior muscle fatigue leads to changes in tibial axial acceleration after impact when ankle dorsiflexion angles are visually controlled. Hum Mov Sci. 2010;29(4): 567-577. PubMed ID: 20579757 doi:10.1016/j.humov.2010.03.004

14. Mitschke C, Kiesewetter P, Milani TL. The effect of the accelerometer operating range on biomechanical parameters: stride length, velocity, and peak tibial acceleration during running. Sensors. 2018; 18(1):130. doi:10.3390/s18010130

15. Wiggins AJ, Grandhi RK, Schneider DK, Stanfield D, Webster KE, Myer GD. Risk of secondary injury in younger athletes after anterior cruciate ligament reconstruction. Am J Sports Med. 2016;44(7): 1861-1876. PubMed ID: 26772611 doi:10.1177/036354651562 1554 